\title{
Risk scores for predicting type 2 diabetes: using the optimal tool
}

\author{
M. Alssema - D. Vistisen - M. W. Heymans - G. Nijpels • \\ C. Glümer • P. Z. Zimmet • J. E. Shaw • M. Eliasson • \\ C. D. A. Stehouwer - A. G. Tabák • S. Colagiuri • \\ K. Borch-Johnsen - J. M. Dekker • \\ for the DETECT-2 collaboration
}

Received: 28 April 2011 / Accepted: 12 May 2011 /Published online: 10 June 2011

(C) Springer-Verlag 2011

Keywords Epidemiology Prediction · Risk scores ·

Type 2 diabetes

\section{Abbreviation \\ ADDITION Anglo-Danish-Dutch Study of Intensive Treatment in People with Screen Detected Diabetes in Primary Care}

\section{Alssema $(\bowtie) \cdot J$. M. Dekker}

Department of Epidemiology and Biostatistics and the EMGO Institute for Health and Care Research,

VU University Medical Center,

Van der Boechorststraat 7 ,

1081 BT Amsterdam, the Netherlands

e-mail: m.alssema@vumc.nl

D. Vistisen $\cdot$ K. Borch-Johnsen

Steno Diabetes Center A/S,

Gentofte, Denmark

\section{W. Heymans}

Department of Methodology and Applied Biostatistics, Institute of Health Sciences, VU University,

Amsterdam, the Netherlands

\section{G. Nijpels}

Department of General Practice and the EMGO Institute for Health and Care Research, VU University Medical Center,

Amsterdam, the Netherlands

\section{Glümer}

Research Centre for Prevention and Health,

The Capital Region of Denmark,

Glostrup, Denmark

\section{P. Z. Zimmet · J. E. Shaw}

Baker IDI Heart and Diabetes Institute,

Melbourne, Victoria, Australia
To the Editor: In a recent commentary related to our paper on the performance of a risk score questionnaire for predicting future diabetes [1], Wareham and Griffin make an interesting point about the effect of real life response rates on the true performance of a risk assessment [2]. In our paper we evaluated the performance of the original Finnish diabetes risk questionnaire to predict future

\author{
M. Eliasson \\ Department of Public Health and Clinical Medicine, \\ Umeå University, Umeå and Sundyby Hospital, \\ Luleå, Sweden \\ C. D. A. Stehouwer \\ Department of Internal Medicine and Cardiovascular \\ Research Institute Maastricht (CARIM), \\ Maastricht University Medical Centre, \\ Maastricht, the Netherlands
}

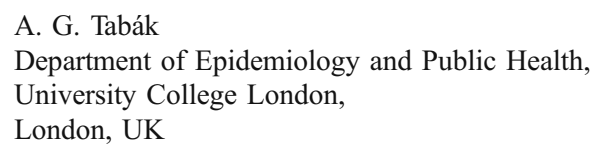

\section{A. G. Tabák}

Semmelweis University Faculty of Medicine, 1st Department of Internal Medicine, Budapest, Hungary

\section{S. Colagiuri} Institute of Obesity, Nutrition and Exercise, University of Sydney, Sydney, NSW, Australia

\section{K. Borch-Johnsen}

Faculty of Health Science, University of Aarhus, Aarhus, Denmark 
screen-detected and clinically diagnosed diabetes. We demonstrated that the performance of the risk score could be improved by adding information on sex, smoking and family history of diabetes [1]. In their commentary, Wareham and Griffin argue that non-response to a questionnaire should be taken into account when evaluating the performance of such a risk questionnaire. They state that when a risk questionnaire is posted out in real life, response rates may be only $50 \%$, and the true sensitivity of the presented score would not be $76 \%$ but rather $38 \%$. They go on to suggest that response rates can be improved to nearly $100 \%$ by using risk scores that are based on data contained in general practice databases, as has been done in the UK $[3,4]$, because they do not require collection of new data. They compare this with the $50 \%$ response rate for risk score questionnaires reported in the Anglo-Danish-Dutch Study of Intensive Treatment in People with Screen Detected Diabetes in Primary Care (ADDITION)-Denmark Study [5], but do not acknowledge that higher response rates have been achieved-for example $78 \%$ in the Hoorn Screening study [6].

The estimated $100 \%$ response rate suggested to be reached by using data from general practice databases ignores several important limitations to using these databases for calculating the risk of diabetes. Missing or inconsistently recorded information is a major problem when using databases. Although in the UK these general practice records have an impressively large amount of recorded data, information on all risk score items is required for calculation of a risk score. Body mass index has been reported to be recorded in approximately $75 \%$ of all persons [4], but information on other risk factors is required in addition to complete most risk scores. One solution to the problem of missing data is to remove one or more items from originally well-performing risk scores [7]. Ignoring risk score items that have a large proportion of missing data or data that are inconsistently recorded will reduce the predictive ability of a risk score and would need re-validation and calibration of the score. Another solution is to tag the person with missing data for opportunistic recording of the missing data at a later visit [7]. The effectiveness of this approach is unknown, but will not reach people who do not visit their general practitioner regularly. Furthermore, risk factors change over time and general practice information will need to be regularly updated. Another major barrier is that globally most healthcare systems do not have computerised primary practice and thus the capacity for central calculation of a risk score. For all these reasons the response rate will be well short of the $100 \%$ suggested by Wareham and Griffin even in situations where this method is an option.
In step-wise screening programmes, loss to follow-up is not only confined to the risk assessment step, irrespective of whether the risk score tool requires completion of a new form or is populated by data contained in a database, but also to when high-risk individuals are invited for further testing, for example (random) glucose measurement. In this second step, roughly similar response rates have been reported for those identified by self-report questionnaire or those identified in general practice records. Documented response rates vary from $77 \%$ in the ADDITION-Denmark trial [5] to $87 \%$ in the Hoorn Screening Study in the Netherlands [6] for those identified by self-assessed risk compared with $74 \%$ for those identified by general practice information [7].

There are a number of important considerations when screening for high risk of future diabetes in order to implement preventive interventions. Our paper focussed on the performance of the risk tool and we have shown that predictive ability of an existing popular risk tool can be improved with minor modifications. The commentary by Wareham and Griffin is a valuable reminder to take non-response in public health programmes seriously. Dealing with non-response is a major challenge in prevention programmes. It is critical that risk assessment has widespread reach to the target population. Many factors influence this process and further effort and research is required to maximise individual and community participation. Using information from general practice records is one approach, but is not the universal answer to this problem. The solution is not a choice between axes or spades but rather the use of both sharp axes and good spades.

Acknowledgements All authors have contributed significantly to design and critical revision of the manuscript and gave their final approval. G.N., J.E.S., S.C., J.M.D. and M.A. drafted the manuscript.

Duality of interest The authors declare that there is no duality of interest associated with this manuscript.

\section{References}

1. Alssema M, Vistisen D, Heymans MW et al (2011) The Evaluation of Screening and Early Detection Strategies for Type 2 Diabetes and Impaired Glucose Tolerance (DETECT-2) update of the Finnish diabetes risk score for prediction of incident type 2 diabetes. Diabetologia 54:1004-1012

2. Wareham NJ, Griffin S (2011) Risk scores for predicting type 2 diabetes: comparing axes and spades. Diabetologia 54:994-995

3. Rahman M, Simmons RK, Harding AH, Wareham NJ, Griffin SJ (2008) A simple risk score identifies individuals at high risk of developing type 2 diabetes: a prospective cohort study. Fam Pract 25:191-196 
4. Hippisley-Cox J, Coupland C, Robson J, Sheikh A, Brindle P (2009) Predicting risk of type 2 diabetes in England and Wales: prospective derivation and validation of QDScore. BMJ 338:b880

5. Christensen JO, Sandbaek A, Lauritzen T, Borch-Johnsen K (2004) Population-based stepwise screening for unrecognised type 2 diabetes is ineffective in general practice despite reliable algorithms. Diabetologia 47:1566-1573
6. Spijkerman AM, Adriaanse MC, Dekker JM et al (2002) Diabetic patients detected by population-based stepwise screening already have a diabetic cardiovascular risk profile. Diabetes Care 25:17841789

7. Sargeant LA, Simmons RK, Barling RS et al (2010) Who attends a UK diabetes screening programme? Findings from the ADDITIONCambridge Study. Diabet Med 27:995-1003 